\title{
Ergodic Cobweb Chaos
}

\author{
AKIO MATSUMOTO* \\ Department of Economics, Niigata University, 8050 2-No-Cho Ikarashi, Niigata 950-21, Japan
}

(Received 9 October 1996)

\begin{abstract}
This study augments the traditional linear cobweb model with lower and upper bounds for variations of output. Its purpose is to detect the relationship between the output constraints and the dynamics of the modified model. Due to the upper and lower bounds, a transitional function takes on a tilted $z$-profile having three piecewise segments with two turning points. It prevents the price (or quantity) dynamics from explosive oscillations. This study demonstrates, by presenting numerical examples, that the modified cobweb model can generate various dynamics ranging from stable periodic cycles to ergodic chaos if a product of the marginal propensity to consume and the marginal product is greater than unity.
\end{abstract}

Keywords: Ergodic chaos, Cobweb dynamics, Quality constraints

JEL: C96, D21, E12, E32

\section{INTRODUCTION}

This study investigates the traditional cobweb model with upper and lower bounds for output variations. Its purpose is to consider implications of the quantity constraints on dynamic behavior of the agricultural economy. The traditional cobweb model, which has naive expectations and linear demand and supply curves, can produce only three types of dynamics: convergence to an equilibrium, convergence to period-2 cycles or divergence. None of these types, however, is satisfactory to explain observed irregular fluctuations of the agricultural goods. Neither the first type nor the third is consistent with observed ups and downs in real economic data, and the second, which implies persistent oscillations in price and quantity, depends on the unrealistic and shaky condition (i.e., supply and demand have exactly the same elasticities). To overcome those limitations, the traditional cobweb model had been modified to produce more realistic dynamics with the help of new developments in non-linear economic dynamics.

\footnotetext{
*E-mail: eakio@hle.niigata-u.ac.jp.
} 
Modifications fall into two groups. In the first of these, we have endogenous non-linear cobweb models in which the supply and/or demand curve are non-linear. The resulting transition functions are chaotic maps having either one turning point or two. Several stability results have been established, which show chaotic oscillations as well as stable periodic cycles (see, Jensen and Urban, 1984; Chiarella, 1988; Finkenstädt and Kuhbier, 1992; Hommes, 1991; 1994). In the second, we find the linear cobweb model taking account of upper bound for variations of output. The upper bound prevents the price (or quantity) dynamics from explosive oscillations. The resulting one-dimensional control map is similar to an expansive tent map (i.e., its slopes are steeper than unity in absolute value). Consequently, it can be shown that the linear cobweb model with upper bound generates not only topological (Li-Yorke) chaos but also ergodic chaos (see, Cugno and Montrucchio, 1980; Nusse and Hommes, 1990). This model, however, has a possibility that almost all trajectories escape from an economically meaningful region for some parameter constellations. In such a case, trajectories are un-bounded and thus it is unable to track the price-quantity evolution.

This study extends the second approach. Returning to the original spirits of "flexible constraints" in Day (1980), it constructs the linear cobweb model augmented with the lower bound for variations of output as well as the upper bound. It purposes to detect the relationship between the output constraints and the dynamics of the model. In particular, by presenting numerical examples, it demonstrates that the modified cobweb model can generate a wide spectrum of dynamic behavior ranging from stable periodic cycles to ergodic chaos.

This study is organized as follows. Section 2 constructs the linear cobweb model with upper and lower bounds for output variations. Section 3 simulates the model. Section 4 makes concluding remarks.

\section{THE COBWEB MODEL WITH FLEXIBLE CONSTRAINTS}

The traditional cobweb model is made up of the following four equations in discrete time:

$$
\begin{aligned}
q_{t}^{\mathrm{d}} & =D\left(p_{t}\right), & & \text { Demand, } \\
q_{t}^{\mathrm{s}} & =S\left(p_{t}^{\mathrm{e}}\right), & & \text { Supply, } \\
q_{t} & =q_{t}^{\mathrm{d}}=q_{t}^{\mathrm{s}}, & & \text { Temporary equilibrium, } \\
p_{t}^{\mathrm{e}} & =p_{t-1}, & & \text { Naive expectation, }
\end{aligned}
$$

where $p_{t}, p_{t}^{\mathrm{e}}, q_{t}^{\mathrm{d}}$ and $q_{t}^{\mathrm{s}}$ are the actual price, the expected price, the quantity demanded and the quantity supplied, respectively, where all are taken for period $t$. This model can be reduced to a onedimensional difference equation

$$
q_{t+1}=S\left(D^{-1}\left(q_{t}\right)\right) \text {. }
$$

In a simple version of the cobweb model, the demand function as well as the supply function is monotonic and thus the composite map, $S\left(D^{-1}\right.$ $\left.\left(q_{t}\right)\right)$, is also monotonic. The slope of $S\left(D^{-1}\left(q_{t}\right)\right)$ evaluated at the equilibrium point characterizes dynamics, since it equals an eigenvalue of the dynamic equation. As long as the slope lies between 0 and $-45^{\circ}$, the eigenvalue in absolute value is less than unity, and thus the equilibrium point is stable. The stable trajectories of price or quantity converge to a stationary state, which does not go with the price or quantity dynamics observed in the real-world. As the slope steepens beyond $-45^{\circ}$, the eigenvalue in absolute value is greater than unity and thus the equilibrium point is unstable. The unstable trajectories explosively oscillate, which also contradicts the actual dynamic behavior. When the slope of $S\left(D^{-1}\left(q_{t}\right)\right)$ is equal to $-45^{\circ}$, period-2 cycles can appear. However, the regular cycles are unlike the irregular nature of the actual cycles. Thus such a simple cobweb model has difficulties to explain cyclical or erratic movements observed in statistical data of agricultural goods. ${ }^{1}$

\footnotetext{
${ }^{1}$ See, for example, the actual fluctuations in agricultural goods provided by Finkenstädt and Kuhbier (1992).
} 
In the real world, it is not surprising that a competitive firm prevents output tomorrow from changing drastically from output today, taking account of capacity constraints, financial constraints, high costs for changing production levels, demand uncertainty, etc. The intention of this study is, in recognition of this fact, to look for an alternative modeling of a firm's behavior that may explain the appearance of cobweb fluctuations. Returning to the original spirits of "flexible coefficient" in Day (1980), ${ }^{2}$ we incorporate cautious behavior of a competitive firm which puts upper and lower bounds on its growth rate. Our specification of the model is as follows:

$$
\begin{aligned}
S\left(p_{t-1}\right) & =-a+b p_{t-1}, & & a>0, \quad b>0, \\
D^{-1}\left(q_{t}\right) & =c-d q_{t}, & & c>0, \quad d>0, \\
q_{t+1} \leq q_{t+1}^{\mathrm{U}} & =(1+\alpha) q_{t}, & & \alpha>0, \\
q_{t+1} \geq q_{t}^{\mathrm{L}} & =(1-\beta) q_{t-1}, & & 1>\beta>0,
\end{aligned}
$$

where the last two equations imply that the growth rate constraints have the effect of preventing output in period $t+1$ from increasing by more than $100 \alpha \%$ or decreasing by less than $100 \beta \%$ from the output of period $t$. Alternatively put, the upper bound and lower bound on the growth rate are, respectively, $\alpha$ and $\beta$. As a result of these bounds, the dynamic system of $q_{t}$ becomes a piecewise linear map:

$$
f\left(q_{t}\right)= \begin{cases}(1+\alpha) q_{t} & \text { for } q_{t} \leq Q_{\mathrm{M}} \\ b c-a-d b q_{t} & \text { for } Q_{\mathrm{M}} \leq q_{t} \leq Q_{\mathrm{m}} \\ (1-\beta) q_{t} & \text { for } q_{t} \geq Q_{\mathrm{m}}\end{cases}
$$

where $Q_{\mathrm{M}}$ and $Q_{\mathrm{m}}$ are a local maximizer and a local minimizer. These are calculated, respectively, as

$$
Q_{\mathrm{M}}=\frac{b c-a}{1+\alpha+b d} \quad \text { and } \quad Q_{\mathrm{m}}=\frac{b c-a}{1-\beta+b d}
$$

Under the assumptions of positive parameters, $b d>0, b c-a>0, \alpha>0$, and $\beta>0$, the map has the tilted- $z$ profile and its non-linearity becomes more pronounced as $b d$ gets larger.

Let $q^{*}$ be a stationary state satisfying $q^{*}=f\left(q^{*}\right)$ (i.e., $q^{*}=(b c-a) /(1+b d)$ ). If $b d>1$, it is oscillatory unstable and forces trajectories to move away in its neighborhood. But fluctuations are bounded by the upper and/or lower constraints of output and thus perpetuated. In order to explain the limiting behavior of bounded fluctuations, we identify three cases that depend on the relative magnitudes between $\alpha$ and $\beta$ : (1) $(1+\alpha)(1-\beta)=1 ;(2)(1+\alpha)(1-\beta)<1 ;$; $)$ $(1+\alpha)(1-\beta)>1$. The corresponding profiles of $f\left(q_{t}\right)$ are depicted, respectively, in Figs. 1(a)-(c) where a cone spanned by the upper and lower constraints is symmetric with respect to $45^{\circ}$ line in the first case and asymmetric in the second and third cases. Considerable progress is found in the study on a map with two piecewise linear segments and a single kinked point like a tent map. However, only the partial results are

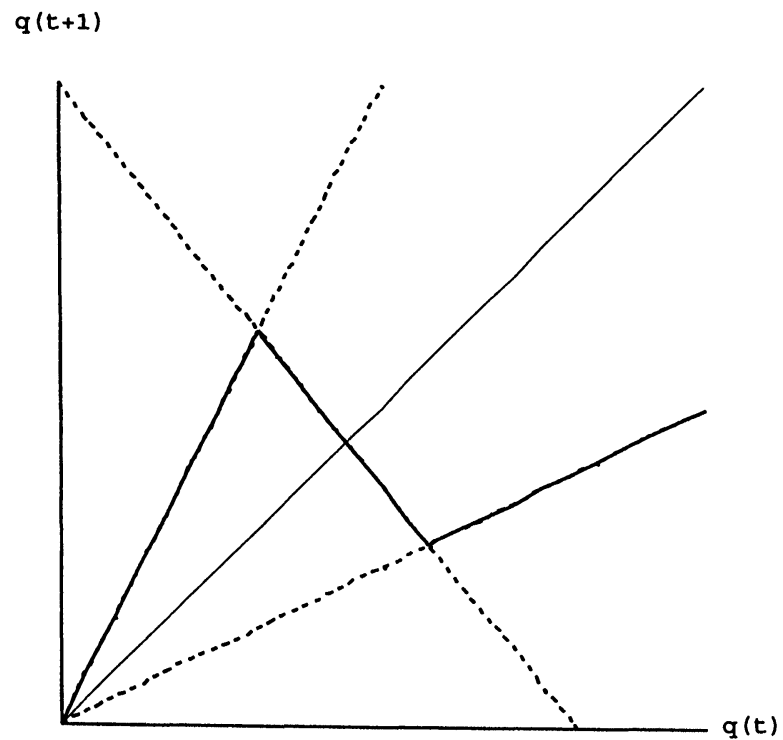

FIGURE 1(a) $(1+\alpha)(1-\beta)=1$. Three profiles of the dynamic equation, $f(q)$.

\footnotetext{
${ }^{2}$ See Day (1980, p. 197) who considers the symmetric upper and lower constraints.
} 


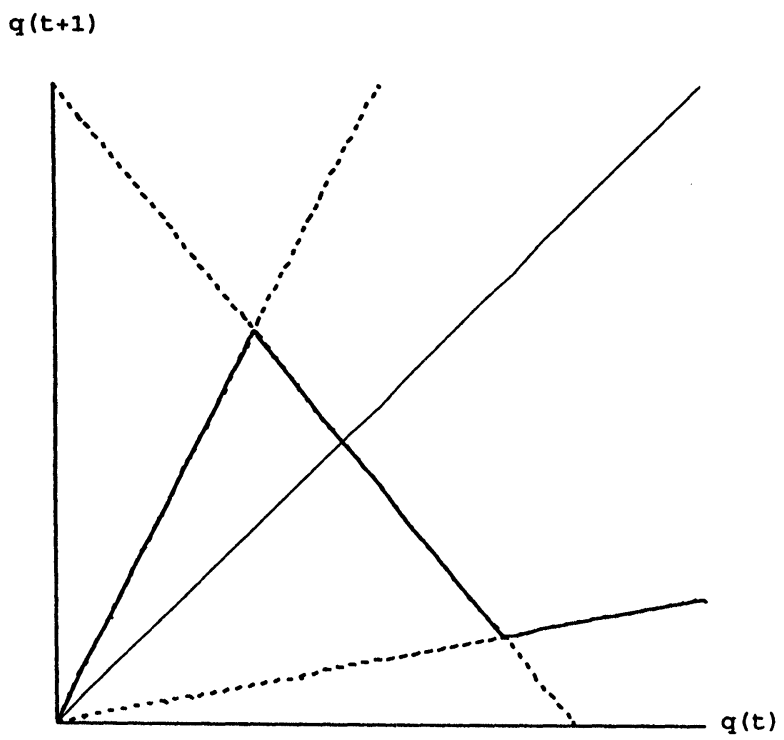

FIGURE 1(b) $(1+\alpha)(1-\beta)<1$.

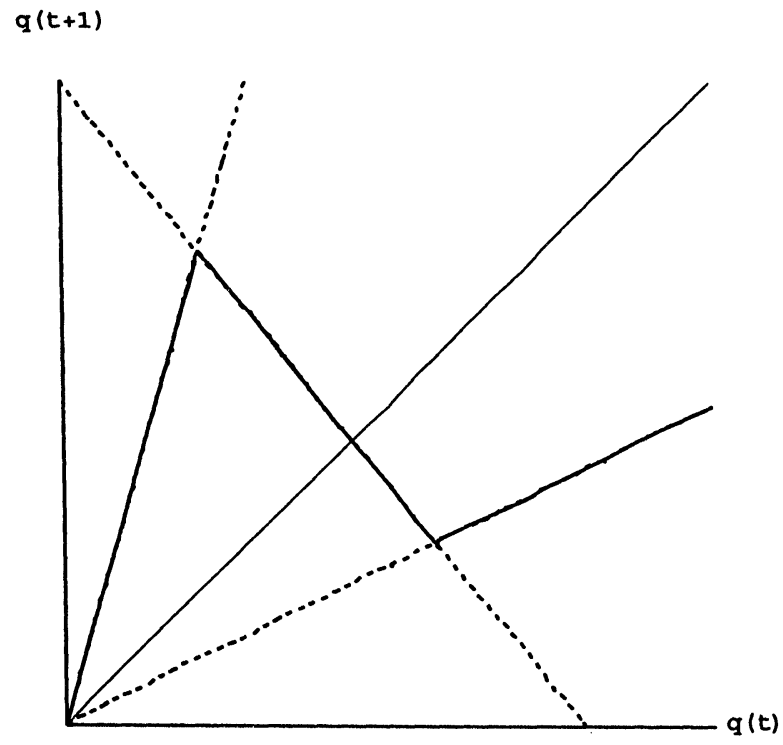

FIGURE 1(c) $(1+\alpha)(1-\beta)>1$.

obtained in the study on a map with three piecewise linear segments and two kinked points like Eq. (2). ${ }^{3}$ Here, simulating the model under different values of $b d(>1)$, we investigate the dynamic structure of the modified cobweb model under each of these three parameter constellations for $\alpha$ and $\beta$.

\section{SIMULATION OF THE MODEL}

To explore the dynamic behavior of $q$, we simulate the model under different.values of $b d$ where $b$ is the marginal propensity to consume and $d$ is the marginal product with respect to the price. We focus on those cases in which oscillations are persistent (i.e., $b d>1$ ). Since the dynamic equation, $f(q)$, has the upper and lower bounds, it induces any trajectories, which are repelled by the unstable equilibrium, bounce back to a neighborhood of the equilibrium point. As it turns out, a combination of $\alpha$ and $\beta$ characterizes asymptotic behavior of trajectories.

\subsection{Symmetric Case}

In this subsection, we deal with the symmetric case in which $(1+\alpha)(1-\beta)=1$ holds. It is numerically and analytically verified that the symmetric-constrained cobweb model can generate stable periodic orbit with period-2.

Two bifurcation diagrams are shown if Fig. 2 . Simulations are performed under the same initial point, $q_{0}=0.1$, and different combinations of $\alpha$ and $\beta: \alpha=1$ and $\beta=0.5$ in Fig. 2(a) and $\alpha=4$ and $\beta=0.8$ in Fig. 2(b). The bifurcation parameters $1 / b d$ is varied in decrement of 0.025 from one to zero (or $b d$ increases from one to infinity). For each values of $1 / b d, f\left(q_{t}\right)$ is iterated 200 times. Although the last 100 iterates are plotted on the vertical axis, only two points are seen in the bifurcation diagrams.

These numerical examples suggest that the symmetric-constrained cobweb model generates only period- 2 cycles. This is analytically verified and summarized as follows.

\footnotetext{
${ }^{3}$ See Day and Shafer (1987).
} 


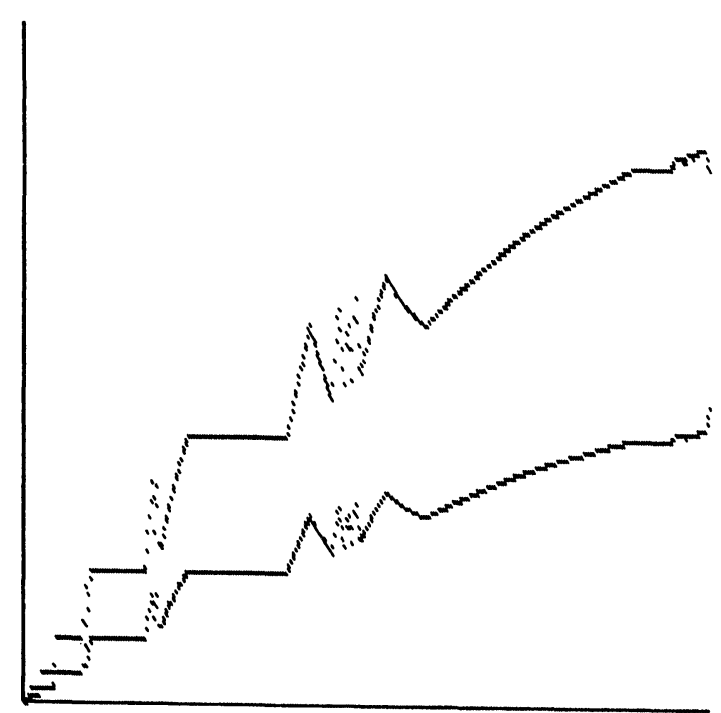

FIGURE 2(a) $\alpha=0.1$ and $\beta=0.5$.

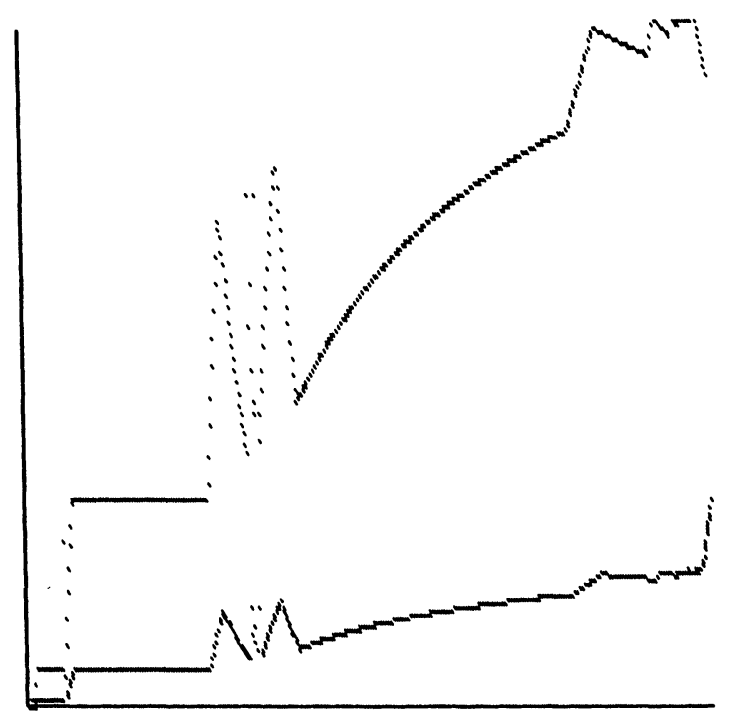

FIGURE 2(b) $\alpha=4$ and $\beta=0.8$. Bifurcation diagrams in the symmetric case.

THEOREM 1. Given $b d>1$, the symmetric-constrained cobweb model generates only stable period-2 cycles.

Proof Let us denote a local minimum of $q$ by $Q^{\min }:=f\left(Q_{\mathrm{m}}\right)$ and a local maximum by $Q^{\mathrm{MAX}}:=f\left(Q_{\mathrm{M}}\right)$. Since $Q^{\text {min }}<Q_{\mathrm{M}}<Q_{\mathrm{m}}<Q^{\mathrm{MAX}}$ holds in the symmetric case, we can divide an interval, $\left[Q^{\mathrm{min}}, Q^{\mathrm{MAX}}\right]$ of $q$ into two subintervals, $A_{0}:=\left[Q^{\text {min }}, Q_{\mathrm{M}}\right]$ and $A_{1}:=\left[Q_{\mathrm{m}}, Q^{\mathrm{MAX}}\right]$. Then it can be checked that $f\left(A_{0}\right)=A_{1}, f\left(A_{1}\right)=A_{0}$. Since $f^{2}(q)=(1+\alpha)(1-\beta) q$ on $A_{i}$ where $(1+\alpha)(1-\beta)=1$ is assumed in the symmetric case, a restriction of $f^{2}(q)$ to $A_{i}$ is the identity map on $A_{i}(i=0,1)$. This means that an orbit $\{q$, $f(g)\}$ for any $q \in A_{i}$ is a period-2 cycle. Since $f^{\prime}=|-b d|>1$ by (2), there is a finite $k$ such that the $k$ th iteration, $f^{k}\left(q_{0}\right) \in A_{0} \cup A_{1}$ for any $q_{0} \in\left(Q_{\mathrm{M}}, Q_{\mathrm{m}}\right)-\left\{q^{*}\right\}$. By the same token, there is a $k^{\prime}$ such that $f^{k^{\prime}}\left(q_{0}\right) \in A_{0} \cup\left(Q_{\mathrm{M}}, Q_{\mathrm{m}}\right)$ if $q_{0}$ $<Q^{\min }$ or $f^{k^{\prime}}\left(q_{0}\right) \in A_{1} \cup\left(Q_{\mathrm{M}}, Q_{\mathrm{m}}\right)$ if $q_{0}>Q^{\mathrm{MAX}}$. Thus every trajectory emanating form a complementary interval of a union of $A_{0}, A_{1}$ and $q^{*}, \overline{A_{0} \cup A_{1} \cup\left\{q^{*}\right\}}$, enters into $A_{0} \cup A_{1}$ after finite iterations. Therefore, we have stable period-2 cycles in the symmetric case.

\subsection{Lower-Asymmetric Case}

In this subsection, we deal with the asymmetric case in which $(1+\alpha)(1-\beta)<1$ holds. As seen in Fig. 1(b), the lower constraint line deviates from the $45^{\circ}$ line more than the upper constraint line so that we call this case lower-asymmetric. Both of the upper and lower bounds are not necessarily effective in the lower-asymmetric case. Only the upper bound is effective for smaller deviations of $b d$ from unity and so are both of the bounds for larger deviations. The dynamics generated by $f\left(q_{t}\right)$ which is constrained by the upper-bound is qualitatively different from the one by $f\left(q_{t}\right)$ which is by the upper and lower bounds. We call the former the one-kinked dynamics and the latter the two-kinked dynamics. In Figs. 3(a) and (b) below, two numerical simulations are performed for the lower-asymmetric conditions; $\alpha=0.25$ and $\beta=0.4$ and $\alpha=2$ and $\beta=0.8$. In both bifurcation diagrams, complex dynamics is observed. As is seen shortly after, the vertical dot line in each of Fig. 3 passes through a critical value of $1 / b d$ that separates the one-kinked dynamics from the two-kinked 


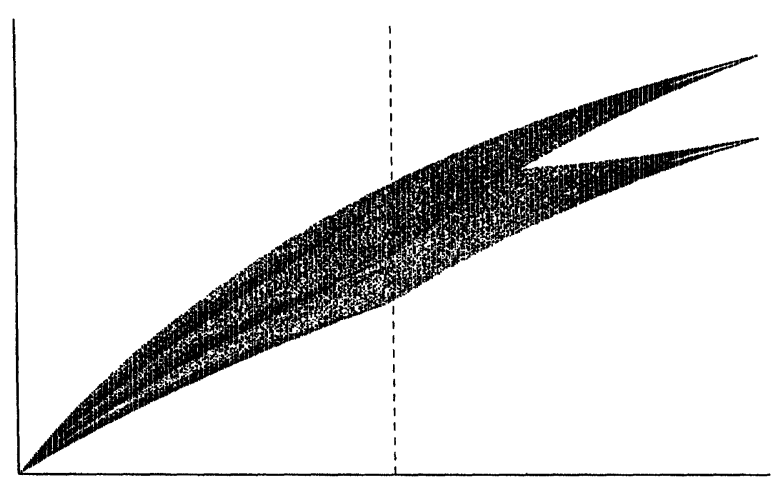

FIGURE 3(a) $\quad \alpha=0.25$ and $\beta=0.4$.

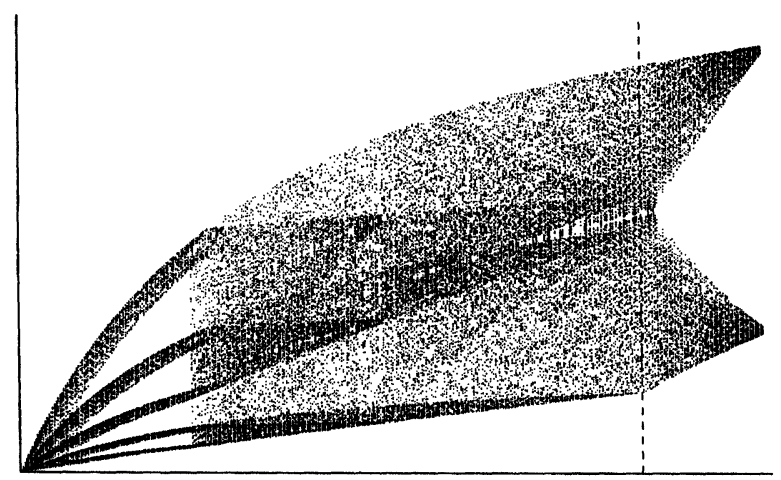

FIGURE $3 \alpha=2$ and $\beta=0.8$. Bifurcation diagrams in the lower asymmetric case.

dynamics. In the following, we confine attention, first, to a case of the one-kinked dynamics in which oscillations are perpetuated due to the upper bound, and then to a case of two-kinked dynamics due to both of the upper- and lowerbounds.

Since a trapping interval eventually traps all trajectories, a restriction of $f(q)$ to the trapping interval governs the asymptotic behavior of $q$. In the lower-asymmetric case, two distinct trapping intervals can be identified which depend on the relation between maximum $Q^{\mathrm{MAX}}$ and minimizer $Q_{\mathrm{m}}$. One trapping interval is defined in a case where $Q^{\mathrm{MAX}} \leqq Q_{\mathrm{m}}$ and the other where $Q^{\mathrm{MAX}}>Q_{\mathrm{m}}$. In particular, subtracting $Q_{\mathrm{m}}$ from
$Q^{\mathrm{MAX}}$ yields

$$
\begin{aligned}
Q^{\mathrm{MAX}}-Q_{\mathrm{m}}= & \frac{(b c-a) \alpha}{(1+\alpha+\sigma)(1-\beta+\sigma)} \\
& \times\left[\sigma-\frac{1+\alpha}{\alpha} \beta\right],
\end{aligned}
$$

where $\sigma:=b d$ for notational simplicity. Since the lower asymmetric condition, $(1+\alpha)(1-\beta)<1$, is transformed into $\alpha /(1+\alpha)<\beta$, there is a $\sigma>1$, denoted by $\sigma_{\mathrm{L}}$, such that $\sigma_{\mathrm{L}}(\alpha /(1+\alpha))=\beta{ }^{4}$ Then Eq. (3) implies the following relations between $Q^{\mathrm{MAX}}$ and $Q_{\mathrm{m}}$ :

$$
\left\{\begin{array}{l}
Q^{\mathrm{MAX}} \leqq Q_{\mathrm{m}} \quad \text { for } \sigma \leqq \sigma_{\mathrm{L}} \\
Q^{\mathrm{MAX}}>Q_{\mathrm{m}} \quad \text { for } \sigma>\sigma_{\mathrm{L}}
\end{array}\right.
$$

Let $V_{1}:=\left[f\left(Q^{\mathrm{MAX}}\right), Q^{\mathrm{MAX}}\right]$ where $f\left(Q^{\mathrm{MAX}}\right)=$ $b c-a-\sigma Q^{\mathrm{MAX}}$. It is the trapping interval for $1<\sigma \leqq \sigma_{\mathrm{L}}$. Namely, whichever point a trajectory starts with, it will enter the interval, $V_{1}$, after finite iterations and stay there afterwards. Furthermore, trajectories inside $V_{1}$ are constrained only by the upper bound. The asymptotic dynamics are governed by the restriction of $f\left(q_{t}\right)$ to $V_{1}$ :

$$
f_{\mid V_{1}}\left(q_{t}\right)=\min \left\{(1+\alpha) q_{t}, b c-a-\sigma q_{t}\right\} .
$$

The conditions, $1+\alpha>1$ and $\sigma>1$, imply that the restricted map $f_{\mid V_{1}}\left(q_{t}\right)$ is expansive and thus has an absolutely continuous invariant measure. ${ }^{5}$ In order to characterize the dynamics generated by $f_{\mid V_{1}}\left(q_{t}\right)$, we rewrite the dynamic Eq. (5) by using two parameters, $A:=(1+\alpha)$ and $B:=\sigma$, in such a way that

$$
\begin{aligned}
& f_{\mid V_{1}}(q) \\
& =\left\{\begin{array}{c}
A q \text { for } q \in V_{1 \mathrm{~L}}:=\left[f\left(Q^{\mathrm{MAX}}\right), Q^{\mathrm{M}}\right] \\
-B q+Q^{\mathrm{M}}(A+B) \\
\text { for } q \in V_{1 \mathrm{R}}:=\left[Q^{\mathrm{M}}, Q^{\mathrm{MAX}}\right] .
\end{array}\right.
\end{aligned}
$$

\footnotetext{
${ }^{4}$ For $\sigma=\sigma^{\mathrm{L}}, Q_{\mathrm{M}}$ is mapped exactly to $Q_{\mathrm{m}}$.

${ }^{5}$ For an expansive map, see Theorem 3 in Day and Pianigiani (1991, p. 45). For dynamic behavior of the expansive map, see Theorem 3 of Day and Schafer (1987, pp. 352-353) and (3) in Property 5 of Nusse and Hommes (1990, p. 13).
} 


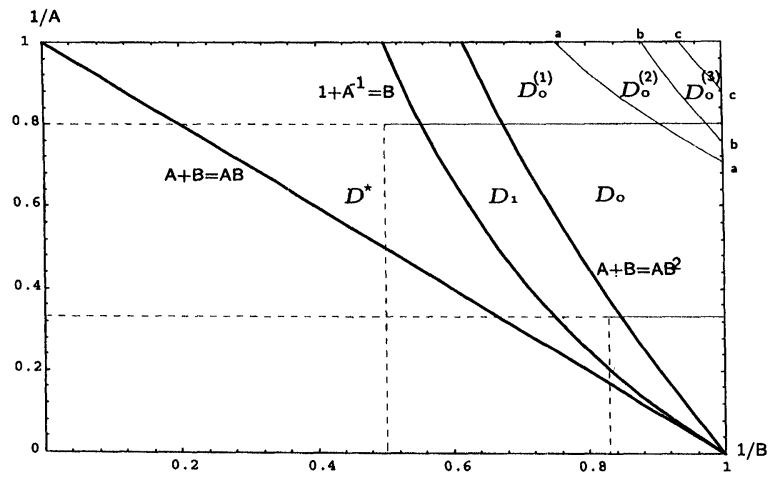

FIGURE 4 Regime classification of two parameters, $A$ and $B$.

Then we will show in Lemma 1 below that $f_{\mid V_{1}}\left(q_{t}\right)$ is equivalent to a unimodal linear map with two parameters $A$ and $B$, denoted by $g_{A, B}(q)$ :

$g_{A, B}(q)= \begin{cases}A q+\frac{A+B-A B}{B} & \text { for } 0 \leqq q \leqq 1-\frac{1}{B}, \\ -B(q-1) & \text { for } 1-\frac{1}{B} \leqq q \leqq 1 .\end{cases}$

LEMMA 1. $f_{\mid V_{1}}\left(q_{t}\right)$ is linearly conjugate to $g_{A, B}(Q)$.

Proof Let

$$
\varphi(q):=\frac{q-f\left(Q^{\mathrm{MAX}}\right)}{Q^{\mathrm{MAX}}-f\left(Q^{\mathrm{MAX}}\right)} .
$$

It can be checked $\varphi(q)$ is a linear isomorphism from $V_{1 i}(i=\mathrm{L}, \mathrm{R})$ onto the unit interval such that $\varphi \circ f_{\mid V_{1}} \circ \varphi^{-1}=g_{A, B}$.

Lemma 1 implies that $f_{\mid V_{1}}(q)$ generates qualitatively the same dynamics as $g_{A, B}(q)$. Ito, Tanaka and Nakada (1979) (ITN henceforth) and Day and Schafer (1987) (DS henceforth) completely characterize the structure of such a map with two piecewise segments and one kinked point (i.e., a map like $g_{A, B}(q)$ ). Thus with the aid of their results, we can characterize the dynamic structure of $f_{\mid V_{1}}(q)$ in the lower-asymmetric case. Figure 4 below reflects the qualitative behavior of $f_{\mid V_{1}}(q)$ for all parameter combinations leading to the expansive map. ${ }^{6}$ Its horizontal axis is $1 / B$ and its vertical axis is $1 / A$. The bifurcation diagram in Fig. 3(a) is obtained by performing simulation for a slice through the $(1 / A, 1 / B)$ space of Fig. 4 for $1 / A=0.8$. Since $\sigma_{\mathrm{L}}=2$ in this example, the switching of the dynamic system form the subsystem with one-kinked point to the one with two-kinked point occurs at $\sigma_{\mathrm{L}}{ }^{-1}=0.5$ in Fig. 4 . The following theorem explains the dynamic behavior observed in the bifurcation diagram of Fig. 3(a) when $\sigma$ is increased from 1 to $\sigma_{\mathrm{L}}$.

THeOrem 2. Given $\alpha=0.25$ and $\beta=0.4$, there exist parameter values, $1<\sigma_{1}<\sigma_{2}<\sigma_{3}<\sigma_{4}<\sigma_{L}$ such that the dynamic system $q_{t+1}=f_{\mid V_{1}}\left(q_{t}\right)$ has a density function of an invariant measure and

(1) its attractor is the union of $2^{3}$ disjoint intervals for $1<\sigma<\sigma_{1}$ where $\sigma_{1} \simeq 1.03$,

(2) its attractor is the union of $2^{2}$ disjoint intervals for $\sigma_{1}<\sigma<\sigma_{2}$ where $\sigma_{2} \simeq 1.16$,

(3) its attractor is the union of 2 disjoint intervals for $\sigma_{2}<\sigma<\sigma_{3}$ where $\sigma_{3} \simeq 1.48$,

(4) its attractor is an interval for $\sigma_{3}<\sigma<\sigma_{4}$ where $\sigma_{4} \simeq 1.8$,

(5) its attractor is an interval for $\sigma_{4}<\sigma<\sigma_{\mathrm{L}}$ where $\sigma_{\mathrm{L}}=2$.

Proof See Theorem 5 for the proof of (1), (2) and (3), and Theorem 6 for the proof of (4) and (5) in Appendix.

According to Theorem 2, the bifurcation scenario of this numerical example is as follows. When $\sigma$ is increased from unity but less than $\sigma_{1}$, $2^{3}$-periodic chaos appears. This means that there are $2^{3}$ disjoint intervals and every trajectory starting outside these intervals eventually enters into a union of these intervals. Furthermore, the union is a support of an invariant measure of $f_{\mid V_{1}}\left(q_{t}\right)$. Intuitively speaking, the trajectory periodically travels one interval to another but chaotically oscillates within each interval. As $\sigma$ increased further, interval halving bifurcations

\footnotetext{
${ }^{6}$ This is a lower part of Fig. 4 in ITN. Definitions of $D_{0}, D_{1}$ and $D^{*}$ are given in Appendix.
} 
occurs for $(A, B) \in D_{0}$ (i.e., $\left.2^{3} \rightarrow 2^{2} \rightarrow 2^{1}\right) .^{7} D_{1}$ and $D^{*}$ are chaotic region in which the system exhibits erratic behavior. Similarly, the bifurcation diagram in Fig. 3(b) is obtained by performing simulation for a slice through the $(1 / A, 1 / B)$ space for $1 / A=\frac{1}{3}$. The switching value of $\sigma$ in this example is $\sigma_{\mathrm{L}}=1.2$ (i.e., $\sigma_{\mathrm{L}}^{-1} \simeq 0.83$ in Fig. 3(b)).

Next, we consider dynamics for $\sigma>\sigma_{\mathrm{L}}$ at which there is a qualitative change in the dynamics behavior of the system. Let $V_{2}:=\left[Q^{\mathrm{min}}, Q^{\mathrm{MAX}}\right]$. It is, as indicated by the second Eq. in (4), the trapping interval for $\sigma>\sigma_{\mathrm{L}}$. Since the inequality relations, $Q^{\mathrm{min}}<Q_{\mathrm{M}}$ $<Q_{\mathrm{m}}<Q^{\mathrm{MAX}}$, holds, $V_{2}$ contains two kinked points, $Q_{\mathrm{M}}$ and $Q_{\mathrm{m}}$, at each of which the switching of the dynamic system from either $(1+\alpha) q$ or $(1-\beta) q$ to $S\left(D^{-1}(q)\right)$ or vice versa takes place. A restriction of $f\left(q_{t}\right)$ to $V_{2}$ has a tilted $z$ shape:

$$
\begin{aligned}
f_{\mid V_{2}}\left(q_{t}\right)= & \max \left\{(1-\beta) q_{t},\right. \\
& \left.\min \left\{(1+\alpha) q_{t}, b c-a-b d q_{t}\right\}\right\} .
\end{aligned}
$$

Although DS (see Table I at p. 355) make some characterizations for such a map with two kinked points, only a little is known about its qualitative behavior. We present numerical examples to detect the influence of $\sigma$ on the two-kinked dynamics. The bifurcation diagrams left to the vertical dot lines in Fig. 3 explore the two-kinked dynamics generated by $f_{\mid V_{2}}$. In both Figs. 3(a) and (b), chaotic behavior still appears as $\sigma$ is increased from $\sigma_{\mathbf{L}}$. If $\sigma$ is further increased, periodic chaos with five intervals appears in the example of Fig. 3(b). Return maps for $\sigma=2$ and $\sigma=5$ are given in Fig. 5 where both of the upper and lower bounds are effective (i.e., trajectories hit the $q(t+1)$

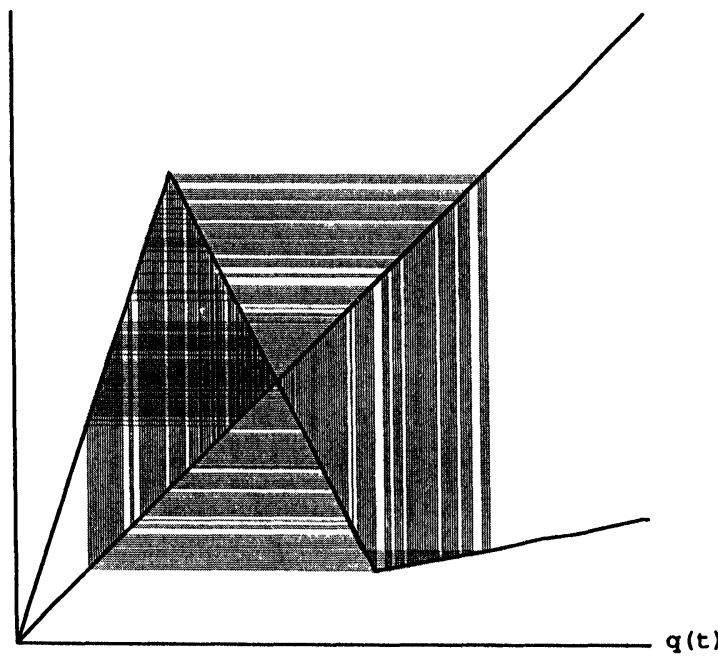

FIGURE 5(a) $\quad \sigma=2$.

$q(t+1)$

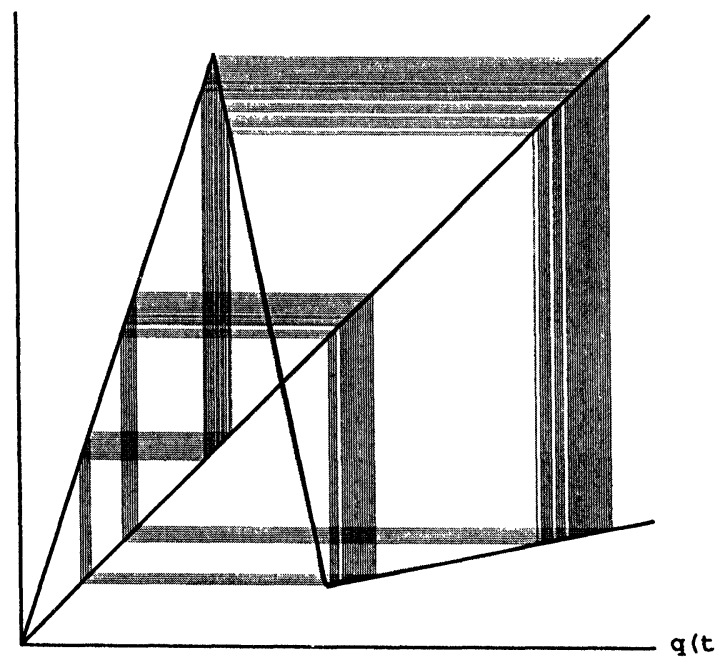

FIGURE $5 \quad \sigma=5$. Return maps of $f(q)$ for $\alpha=2$ and $\beta=0.8$.

\footnotetext{
${ }^{7}$ Interval halving bifurcations in $D_{0}$ proceed as follows. Let $(A, B) \in D_{0}$ and let $A_{0}=\left[g_{A, B}(0), 1\right]$ and $A_{1}=\left[0, g_{A, B}^{2}(0)\right]$. Then we have the following three results: (1) $g_{A, B}\left(A_{i}\right)=A_{j}, i, j=0,1, i \neq j ;(2) g_{A, B \mid A_{j}}^{2}(j=0$ or 1$)$ is linearly conjugate to $g_{B^{2}, A B}$; (3) $(A, B) \in D_{0}^{(1)}$ implies $\left(B^{2}, A B\right) \in D^{*}$ where (1) and (2) are due to Lemma 1.1 of ITN and (3) to Theorem 1.2 (ii) of ITN. $g_{B^{2}, A B}$ has an absolutely continuous invariant measure on $[0,1]$ because it is expansive, and so does $g_{A, B \mid A_{j}}^{2}$ on the interval $A_{j}$ by (2). By (1), there are two intervals in which irregular or unpredictable behavior occurs. We thus have period-2 chaos for $(A, B) \in D_{0}^{(1)}$ by (3). If $(A, B) \in D_{0}^{(2)}$, then Theorem 1.2 (ii) of ITN $\left(B^{2}, A B\right) \in D_{0}^{(1)}$ which then implies $\left(A^{2} B^{2}, A B^{3}\right) \in D^{*}$. Thus for $(A, B) \in D_{0}^{(2)}$, there are $2^{2}$ intervals on which the system is ergoic. By the same token, for $(A, B) \in D_{0}^{(3)}$, there are $2^{3}$ intervals on which the system is ergoic.
} 
bounds and are bounced back to an inside of the trapping interval).

\subsection{Upper-Asymmetric Case}

In this subsection, we deal with the asymmetric case in which $(1+\alpha)(1-\beta)>1$ holds. As seen in Fig. 1(c), the upper constraint line deviates from the $45^{\circ}$ line more than the lower constraint line so that we call this case upper-asymmetric. The following analysis is analogous to that in the lower-asymmetric case. The one-kinked dynamics is considered and then the two-kinked dynamics is followed. The dis-similarity is found in that the dynamics system with one-kinked point is not expansive. We, first, identify two trapping intervals which depend on the relation between minimum $Q^{\text {min }}$ and maximizer $Q_{\mathrm{M}}$ : one where $Q_{\mathrm{M}}<Q^{\mathrm{min}}$ : the other where $Q_{\mathrm{M}}>Q^{\mathrm{min}}$. Subtracting $Q^{\text {min }}$ from $Q_{\mathrm{M}}$ yields

$$
\begin{array}{r}
Q_{\mathrm{M}}-Q^{\min }=\frac{(b c-a) \beta}{(1+\alpha+\sigma)(1-\beta+\sigma)} \\
\times\left[\sigma-\frac{1-\beta}{\beta} \alpha\right] .
\end{array}
$$

Since the upper-asymmetric condition, $(1+\alpha)$ $(1-\beta)>1$, is written as $\beta /(1-\beta)<\alpha$, there is a $\sigma>1$, denoted by $\sigma_{\mathrm{U}}$, such that $\sigma_{\mathrm{U}}(\beta /(1-\beta))$ $=\alpha$. Then the Eq. (7) implies the following relations between $Q_{\mathrm{M}}$ and $Q^{\mathrm{min}}$ :

$$
\begin{cases}Q_{\mathrm{M}} \leqq Q^{\min } & \text { for } \sigma \leqq \sigma_{\mathrm{U}} \\ Q_{\mathrm{M}}>Q^{\min } & \text { for } \sigma>\sigma_{\mathrm{U}}\end{cases}
$$

Let $\quad U_{1}:=\left[Q^{\min }, f\left(Q^{\min }\right)\right] \quad$ where $f\left(Q^{\min }\right)=$ $b c-a-\sigma Q^{\text {min }}$. It is a trapping interval for $1<\sigma \leq \sigma_{\mathrm{U}}$. A restriction of $f(q)$ to $U_{1}$,

$$
f_{\mid U_{1}}(q)=\max \left\{b c-a-\sigma q_{t},(1-\beta) q_{t}\right\},
$$

governs asymptotic dynamics of $q_{t}$. Let us divide the trapping interval, $U_{1}$, into two subintervals,
$U_{1 \mathrm{~L}}:=\left[Q^{\mathrm{min}}, Q_{\mathrm{m}}\right]$ and $U_{1 \mathrm{R}}:=\left[Q_{\mathrm{m}}, f\left(Q^{\mathrm{min}}\right)\right]$. The restricted map, $f_{\mid U_{1}}$, has a slope greater than unity in absolute value on $U_{1 \mathrm{~L}}$ (i.e., $|-\sigma|>1$ ) and less than unity on $U_{1 \mathrm{R}}$ (i.e., $0<1-\beta<1$ ). Namely, it is not expansive. It, however, can exhibit complex dynamics involving chaos. As shown in Lemma 2 below, $f_{\mid U_{1}}$ is linearly conjugate to the unimodal map, $g_{A, B}(q)$. In consequence, we can detect the dynamic behavior by applying the results of ITN to $f_{\mid U_{1}}(q)$. Let $A:=1-\beta$ in the upper-asymmetric case while $B:=\sigma$ as in the lower-symmetric case. The restricted map, $f_{\mid U_{1}}(q)$, can be written as

$$
f_{\mid U_{1}}(q)=\left\{\begin{array}{c}
-B q+Q_{\mathrm{m}}(A+B) \\
\text { for } q \in U_{1 \mathrm{~L}}=\left[Q^{\min }, Q_{\mathrm{m}}\right], \\
A q \quad \text { for } q \in U_{1 \mathrm{R}}=\left[Q_{\mathrm{m}}, f\left(Q^{\mathrm{min}}\right)\right]
\end{array}\right.
$$

Then we have the following lemma.

LEMMA 2. $f_{\mid U_{1}}(q)$ is linearly conjugate to $g_{A, B}(q)$.

Proof Let

$$
\phi(q):=\frac{q-Q^{\min }}{f\left(Q^{\min }\right)-Q^{\min }} .
$$

For $\phi^{-1}(q) \in U_{1 \mathrm{R}}, \quad \phi \circ f_{\mid U_{1}} \circ \phi^{-1}(q)=A q-A / B$ which we denote by $\tau(q)$. Let $h(q)=1-q$. Then $h^{-1} \circ \tau \circ h(q)=A q+(A+B-A B) / B$ which is $g_{A, B}(q)$ for $q \in[1-1 / B, 1]$. Similarly, we can show that for $\phi^{-1}(q) \in U_{1 \mathrm{~L}} f_{\mid U_{1}}(q)$ is equivalent to $g_{A, B}(q)$ for $q \in[0,1-1 / B]$.

Lemma 2 suggests that $f_{\mid U_{1}}(q)$ generates qualitatively the same dynamics as $g_{A, B}(q)$ with $A<1$ and $B>1$. Figure 6 below, which is similar to Fig. 4 in the lower-symmetric case, is a 2parameter bifurcation diagram where the horizontal axis is $1 / B$ and the vertical axis is $1 / A .^{8}$ In Fig. 7, two numerical simulations are performed along the straight line passing through $1 / A=1.2$ and one through $1 / A=1.8$ in Fig. $6 .^{9}$ Theorem 3 below summarizes the results for the dynamics generated by the latter numerical example.

\footnotetext{
${ }^{8}$ Figure 6 is an upper half of Fig. 4 in ITN.

${ }^{9}$ Since $A=1-\beta, \beta=\frac{1}{6}$ in the former example and $\beta=\frac{4}{9}$ in the latter example.
} 


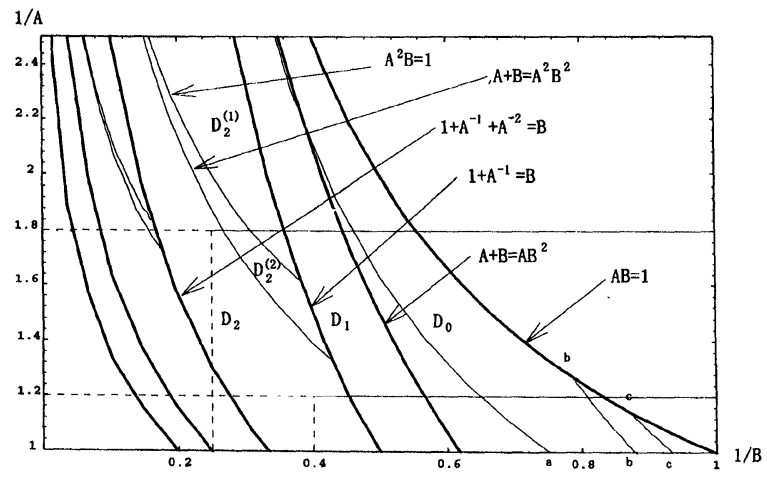

FIGURE 6 Regime classification of parameters $(A, B)$.

THEOREM 3. Given $\alpha=3.2$ and $\beta=4 / 9$, there exist parameter values, $1<\sigma_{1}<\sigma_{2}<\sigma_{3}<\sigma_{4}<\sigma_{5}<\sigma_{\mathrm{U}}$ such that the dynamic system, $q_{t+1}=f_{\mid U_{1}}\left(q_{t}\right)$, has

(1) a period-2 cycle for $1<\sigma<\sigma_{1}$ where $\sigma_{1}=1.8$,

(2) $2^{m}$-periodic chaos $\sigma_{1}<\sigma<\sigma_{2}$ where $\sigma_{2} \simeq 2.25$ and $m=1,2$,

(3) an ergodic chaos for $\sigma_{2}<\sigma<\sigma_{3}$ where $\sigma_{3}=2.8$,

(4) a stable period-3 cycle for $\sigma_{3}<\sigma<\sigma_{4}$ where $\sigma_{4} \simeq 3.24$,

(5) 6-periodic chaos and then 3-periodic chaos for $\sigma_{4}<\sigma<\sigma_{5}$ where $\sigma_{5} \simeq 3.72$,

(6) an ergodic chaos for $\sigma_{5}<\sigma<\sigma_{\mathrm{U}}$ where $\sigma_{\mathrm{U}}=4.0$.

Proof The proof of (1): as $B>1$ and $A B<1$ for $1<\sigma<\sigma_{1}$, it can be checked that there exists a unique periodic orbit with period 2. The proof of (2): as $(A, B) \in D_{0}$, see Theorem 4 in the Appendix. The proof of (3): as $(A, B) \in D_{1}$, see Theorem 5. The proof of (4): as $(A, B)$ $\in D_{2}^{(1)}$, see Theorem 6. The proof of (5): as $(A, B) \in D_{2}^{(2)}$, see Theorem 7. The proof of (6): as $(A, B) \in D_{2}^{*}$, see Theorem 5 .

Figure 7(b) illustrates the dynamics described by Theorem 3. Concerning the bifurcation scenario with respect to $\sigma$, (1)-(3) imply that as $\sigma$ is increased from unity, the stable period- 2 cycle

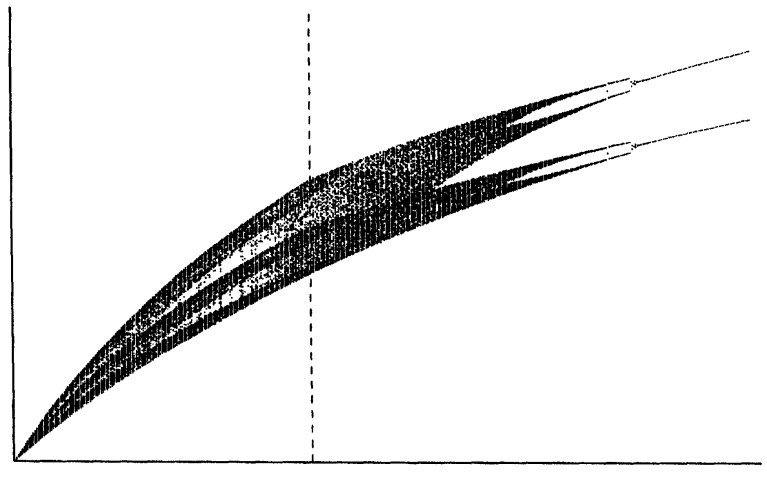

FIGURE 7(a) $\quad \alpha=0.5$ and $\beta=1 / 6$.

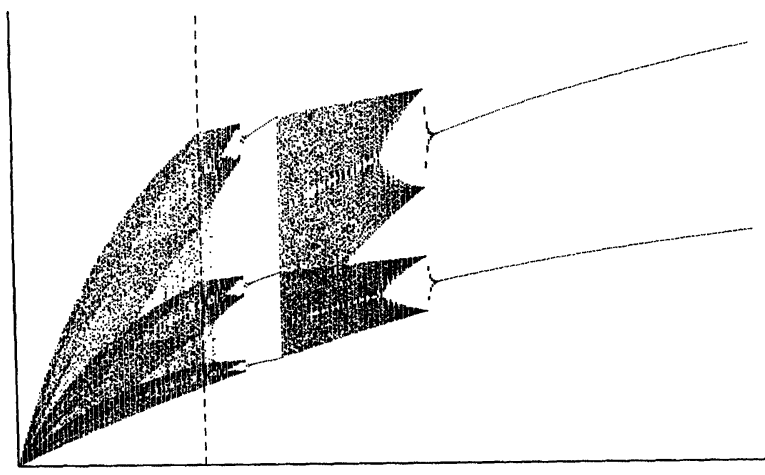

FIGURE $7 \quad \alpha=3.2$ and $\beta=8 / 9$. Two bifurcation diagrams in the upper-symmetric case.

bifurcates to periodic chaos having the union of $2^{\mathrm{m}}$ disjoint intervals as its attactor and then to ergodic chaos with one intervals. In the interval $\left(\sigma_{3}, \sigma_{4}\right)$, there exists a stable period-3 cycle to which almost all trajectories converge. (5) implies that in the interval $\left(\sigma_{4}, \sigma_{5}\right), f_{\mid U_{1}}$ has an absolutely continuous invariant measure. Its support is a union of three disjoint intervals for $\sigma$ close to $\sigma_{5}$ (i.e., 3-periodic chaos) while it is a union of six disjoint intervals for $\sigma$ close to $\sigma_{4} \cdot{ }^{10}$ Finally the ergodic chaos with one interval appears. If $1 / B$ is decreased from unity to zero along the lower straight line for $1 / A=1.2$ in Fig. 6 , the bifurcation scenario proceeds similar to one described by Theorem 2: the equilibrium point bifurcates to

\footnotetext{
${ }^{10}$ See Corollary 3.2 of ITN which gives all the details.
} 
stable period- 2 cycles, periodic chaos with $2^{3}$ intervals, $2^{2}$ intervals, 2 intervals and then one interval.

If $\sigma$ is further increased from $\sigma_{\mathrm{U}}, Q^{\mathrm{min}}<Q_{\mathrm{M}}$ holds. Then the dynamic system is switched from (8) to a restriction of $f\left(q_{t}\right)$ to a trapping interval $U_{2}:=\left[Q^{\min }, Q^{\max }\right]$. It has three line segments and two kinked points, $Q_{\mathrm{M}}$ and $Q_{\mathrm{m}}$. The tilted $z$ shaped restricted map governs the quantity evolution and, according to Fig. 7(a), generates aperiodic fluctuations.

\section{CONCLUDING REMARKS}

This study investigates the dynamic structure of a linear cobweb model with upper and lower bounds for variations on output. Simulating the model under different values of $b d$, we have demonstrated that the modified cobweb model may generate chaotic behavior if the output constraints are asymmetric and that it can generate stable period-2 cycles whose amplitudes depend on the prevailing parameter constellations (i.e., choice of initial point, values of $b d$, etc.) if the output constraints are symmetric. Although the bifurcation diagrams in asymmetric cases imply the chaotic behavior, the bifurcation scenario to chaos is different according to whether the constraints are upper- or lower-asymmetric. In the lower-asymmetric case, the modified cobweb model generates persistent and aperiodic cycles for any values of $b d$ greater than unity. The control system (i.e., a restriction of $f(q)$ to a trapping interval) switches from an expansive map to a tilted $z$-shaped map as $b d$ is increased. In consequence, periodic chaos with disjoint intervals and then truly chaos appear as $b d$ exceeds unity. On the other hand, in the upperasymmetric case, the control system switches from an asymmetric tent map to a tilted $z$-shaped map. A road to chaos in the upper-symmetric case is different from one in the lower-symmetric case. As $b d$ is increased from unity, the quantity dynamics (as well as the price dynamics) move from stable period-2 cycles, to periodic chaos with $2^{2}$ intervals, to one with 2 interval and then to truly chaos (i.e., chaos with 1 interval), the last of which shrinks to zero as $b d$ goes to infinity.

What implications do the simulations have for the cobweb model with the upper and lower bounds? $b d>1$ is necessary for generations of chaos in the asymmetric case. Here $b$ is the marginal propensity to consume which is the decision variables of the demand side while $d$ is the marginal product with respect to price which is the decision variable of the supply side. Thus it can be stated that one source of such complex dynamics involving chaos is an interaction between the consumers and the producers. Furthermore, since the marginal propensity to consume is expected to satisfy condition $0<b<1, b d>1$ requires the strong production response to a change in price (i.e., the large value of $b$ ). This study, therefore, implies that agricultural economies with the strong production response tend to exhibit erratic dynamics of the agricultural goods when the upper and lower bounds are imposed on the growth rate.

\section{Acknowledgement}

I would like to thank K. Higuchi, Y. Sakai, A. Simonovits and M. Sonis for valuable comments and constructive suggestions. The earlier version of the paper benefited from comments received during presentations at the conference on "Comerce, Complexity and Evolution" at University of New South Wales, Sydney, Australia, February 12-13, 1996 and the 5th World Congress of the RSAI at Risshou University, Tokyo, Japan, May 2-6, 1996. I am grateful to financial support from the Nomura Foundation. Needless to say, all remaining errors are my own.

\section{References}

[1] Chiarella, C. (1988). The cobweb model. Its instability and the onset of chaos, Economic Modeling, 5, 377-384.

[2] Cugno, F. and Montrucchio, L. (1980). Some new techniques for modeling nonlinear economic fluctuations: A brief survey, in: R.M. Goodwin, M. Krüger and A. 
Vercelli, Eds., Nonlinear Models of Fluctuating Growth, LNEM 228, Springer-Verlag, Berlin, pp. 146-165.

[3] Day, R. (1980). Cobweb models with explicit suboptimization, in: R. Day and A. Cigno, Eds., Modeling Economic Change: The Recursive Programming Approach, North-Holland, Amsterdam, pp. 191-215.

[4] Day, R. and Hanson, K. (1991). Cobweb chaos, in: T. Kaul and J. Sengupta, Eds., Essay in Honor of Karl A. Fox, Elsevier Science Publishers B.V., pp. 175-192.

[5] Day, R. and Pianigiani, R. (1991). Statistical dynamics and economics, Journal of Economic Behavior and Organization, 16, 37-83.

[6] Day, R. and Schafer, W. (1987). Ergodic fluctuations in deterministic economic model, Journal of Economic Behavior and Organization, 8, 339-361.

[7] Finkenstädt, B. and Kuhbier, P. (1992). Chaotic dynamics in agricultural markets, Annals of Operations Research, 37, 73-96.

[8] Hommes, C. (1991). Adaptive learning and roads to chaos, Economic Letters, 36, 127-132.

[9] Hommes, C. (1994). Dynamic of the cobweb model with adaptive expectations and nonlinear supply and demand, Journal of Economic Behavior and Organization, 24, 315335.

[10] Huang, W. (1995). Caution implies profit, Journal of Economic Behavior and Organization, 27, 257-277.

[11] Ito, S., Tanaka, S. and Nakada, H. (1979). On unimodal linear transformation and chaos II, Tokyo Journal of Mathematics, 2, 241-259.

[12] Jensen, R. and Urban, R. (1984). Chaotic price behavior in a non-linear cobweb model, Economic Letters, 15, 235-240.

[13] Matsumoto, A. (1996). Ergodic chaos in inventory oscillations; an example, Chaos, Solitons and Fractals, 7, $2175-2188$.

[14] Nusse, H. and Hommes, C. (1990). Resolution of chaos with application to a modified Samuelson model, Journal of Economic Dynamics and Control, 14, 1-19.

\section{APPENDIX}

In this Appendix, I recapitulate some results from ITN and DS.

Define a region of parameters $A$ and $B$ :

$$
\begin{aligned}
& D=\{(A, B) \mid B>1, A B>1, A+B \geq A B\}, \\
& D_{0}=\left\{(A, B) \in D \mid g_{A, B}(0) \geq x^{*}\right. \\
& \left.\quad \text { where } x^{*}=g_{A, B}\left(x^{*}\right)\right\},
\end{aligned}
$$

$$
\begin{aligned}
D_{1}= & \left\{(A, B) \in D \mid x^{*}>g_{A, B}(0) \geq y^{*}\right. \\
& \text { where } \left.y^{*} \text { is a maximizer of } g_{A, B}\right\} \\
D^{*}= & \left\{(A, B) \in D \mid y^{*}>g_{A, B}(0)\right. \\
D_{k}= & \left\{(A, B) \in D \mid g_{A}^{k},{ }_{B}(0)>y^{*} \geq g_{A, B}^{k-1}(0)\right\} \\
D_{k}^{(1)}=\left\{(A, B) \in D_{k} \mid A^{k} B \leq 1\right\} & \\
D_{k}^{(2)}=\left\{(A, B) \in D_{k} \mid A^{k} B>1\right. & \left.\quad \text { and } A+B \geq A^{k} B^{2}\right\} \\
D_{k}^{*}= & D_{k}-\left(D_{k}^{(1)} \cup D_{k}^{(2)}\right)
\end{aligned}
$$

THEOREM 4. (Theorem 1.2 and Corollary 3.1 of ITN) For $(A, B) \in D_{0}$, there exist disjoint $2^{m}$ intervals, $A_{0}, A_{1}, \ldots, A_{2^{m}-1}$ such that $g_{A, B}\left(A_{i}\right)=$ $A_{i+1}$ for $0 \leq i \leq 2^{m}-2$ and $g_{A, B}\left(A_{2^{m}-1}\right)=A_{0}$. $g_{A, B}(\cdot)$ has absolutely continuous invariant ergodic measure whose support is equal to $\cup_{i=0}^{2^{m}-1} A_{i}$.

THEOREM 5. (Theorem 3.1. of ITN) For $(A, B) \in$ $D_{1} \cup\left(\cup_{k=2}^{\infty} D_{k}^{*}\right) \cup D^{*}$, there exists a density function of an invariant measure for $g_{A, B}(\cdot)$.

THEOREM 6. (Theorem 2.2. of ITN, the first part of Theorem 5 of $D S)$ For $(A, B) \in D_{k}^{(1)}$, almost all points of $[0,1]$ are asymptotically periodic. When the strict inequality holds, then almost all orbits approach the periodic orbit.

Theorem 7. (Theorem 2.3. and Corollary 3.2 of $I T N$, the second part of Theorem 5 of DS) For $(A B) \in D_{k}^{(2)}$, there exist disjoint $k$ intervals, $J_{0}$, $J_{1}, \ldots, J_{k-1}$ such that $g_{A, B}\left(J_{i}\right)=J_{i+1}$ for $0 \leq i$ $\leq k-1$ and $g_{A, B}\left(J_{k-1}\right)=J_{0} \cdot g_{A, B}(\cdot)$ has absolutely continuous invariant ergodic measure whose support is equal to $\cup_{i=0}^{k} J_{i}$. 


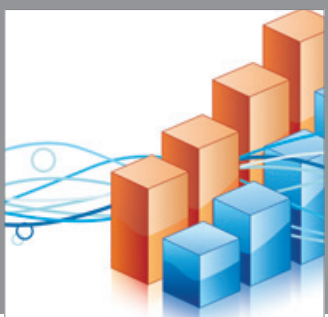

Advances in

Operations Research

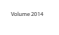

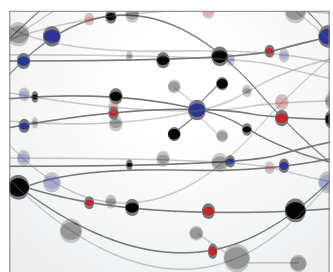

\section{The Scientific} World Journal
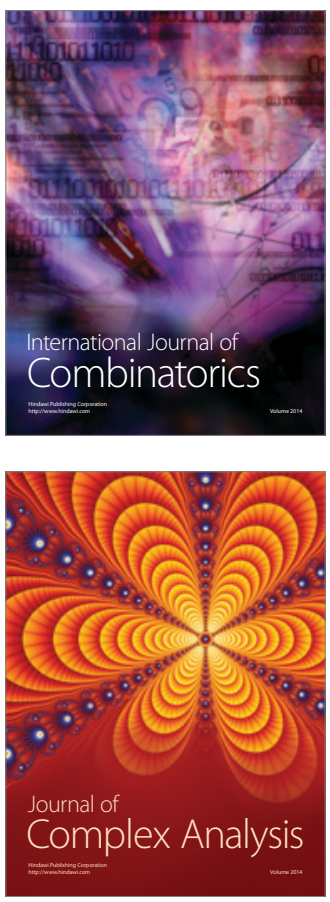

International Journal of

Mathematics and

Mathematical

Sciences
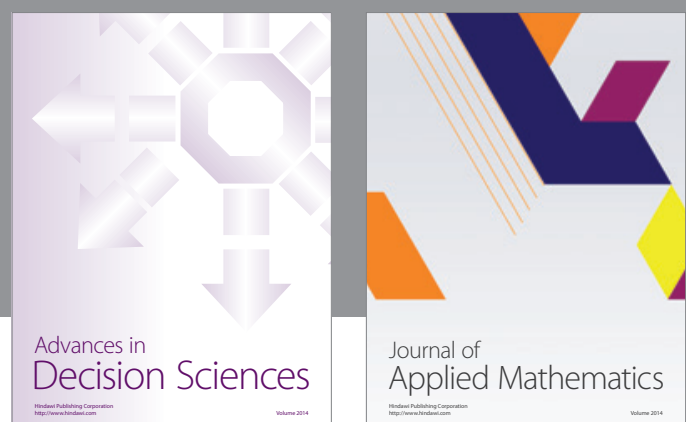

Journal of

Applied Mathematics
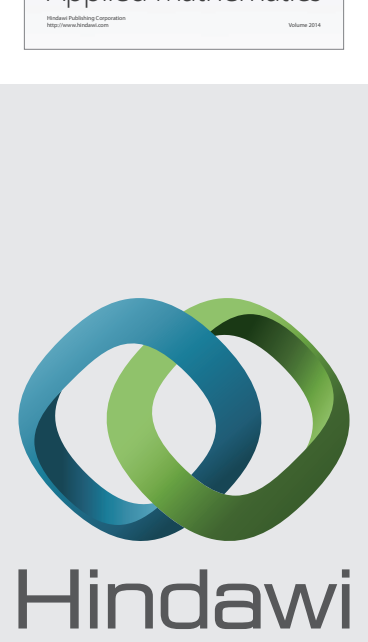

Submit your manuscripts at http://www.hindawi.com
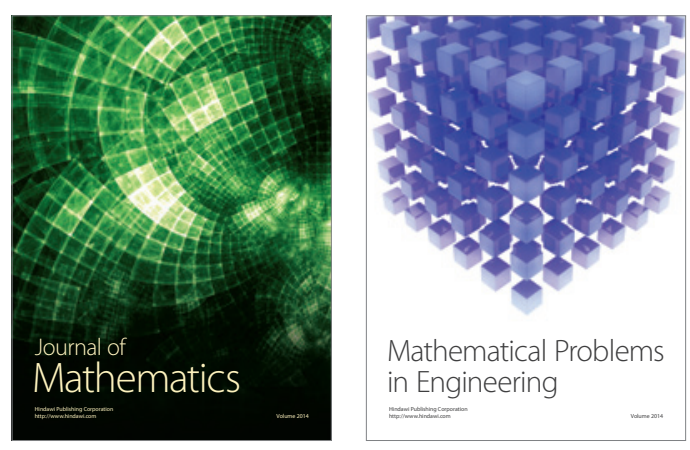

Mathematical Problems in Engineering
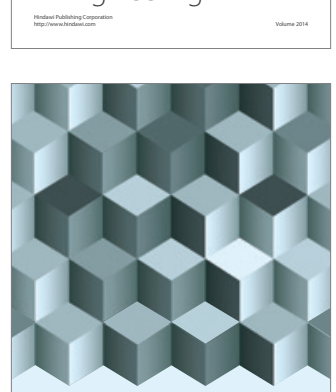

Journal of

Function Spaces
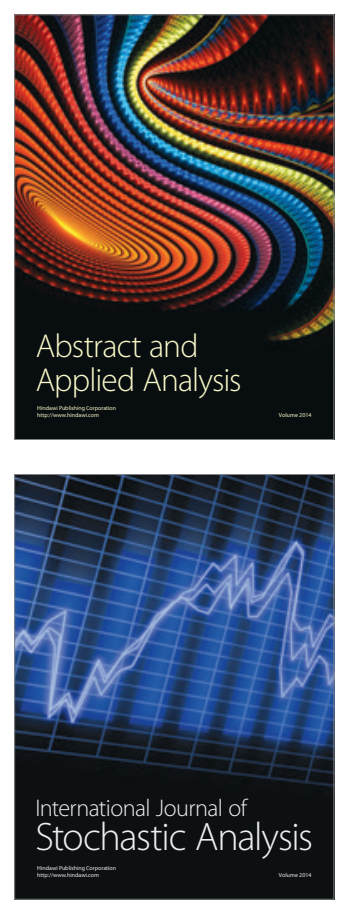

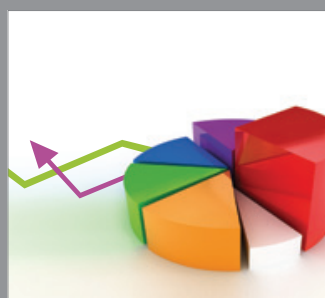

ournal of

Probability and Statistics

Promensencen
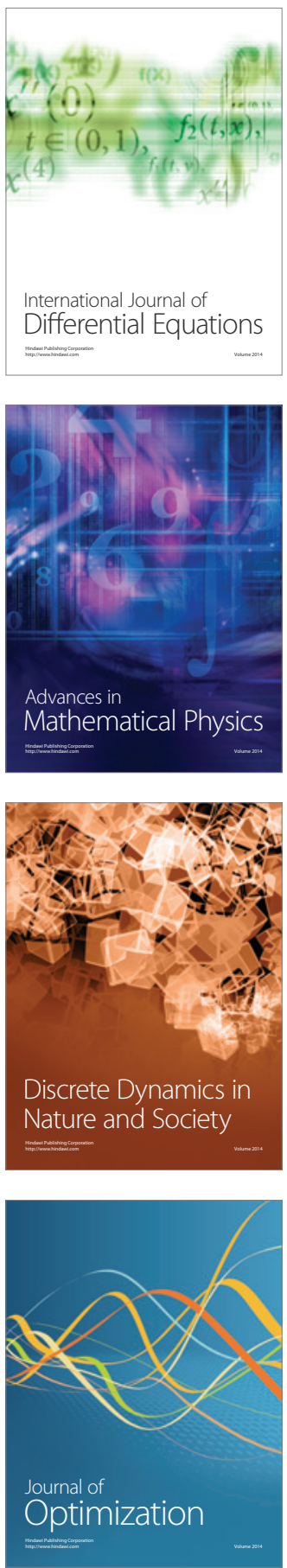